\title{
THE SHORT CIRCUMFERENCE DAMPING RING DESIGN FOR THE ILC
}

\author{
M. Korostelev, F. Zimmermann, CERN, Geneva, Switzerland; \\ K. Kubo, M. Kuriki, S. Kuroda, T. Naito, J. Urakawa, KEK, Ibaraki, Japan; \\ M. Ross, SLAC, Menlo Park, California, USA
}

\section{Abstract}

The ILC damping ring tentative design is driven by the operational scenario of the main linac, the beam-dynamics demand of producing a stable and high-quality beam, the injection/extraction scheme and the kicker performance. In this paper, a short circumference damping ring design based on TME cells is described. The ring accommodates injection kickers which provide a flat top of $280 \mathrm{nsec}$ and a $60 \mathrm{nsec}$ rise and fall time and very fast strip-line kickers for beam extraction with a $2 \mathrm{nsec}$ rise and fall time for $3-\mathrm{MHz}$ operation.

\section{INTRODUCTION}

The original TESLA design featured a 17-km long "dogbone" damping ring [1]. Due to the large circumference, this damping ring must operate with local long-distance "coupling bumps" in order to achieve an acceptable spacecharge tune shift, and it can be sensitive to various types of instabilities and perturbations, such as linac stray fields or ground motion. An advantage of the large circumference are the loose kicker requirements. However, despite of this large circumference the bunches still need to be extracted individually from the damping ring, since the bunch spacing in the $17-\mathrm{km}$ ring of about $20 \mathrm{~ns}$ is much shorter than the 3-ns spacing in the superconducting linac.

Ongoing worldwide studies, e.g., in [2], suggest that it may be possible to build kicker magnets with significantly shorter rise and fall times, of the order of a few ns. Given this possibility of faster kickers for injection and extraction, a ring with a smaller circumference then becomes an attractive option. We here report about one such design, which has a circumference of $3 \mathrm{~km}$.

\section{DESIGN STRATEGY}

The starting point of the damping-ring optimization was the KEK design "TME5.1" of a 3-km ILC damping ring, created by S. Kuroda [3]. This ring design combined TMEcell arcs adopted from the NLC damping ring design with 80 ATF-type wigglers at the center of each arc, and long empty straight sections. The wigglers covered two times a total length of $160 \mathrm{~m}$ and had the ATF wiggler period of 40 $\mathrm{cm}$. The length of the arc bending magnet was $9 \mathrm{~m}$. The ring energy of $5 \mathrm{GeV}$ was chosen as in the TESLA design [6].

The emittance performance of this ring was recently improved by modifying both the wiggler parameters and the arcs. The optimization was based on the expression for the equilibrium emittance without intrabeam scattering for a hard-edged wiggler model [4],

$$
\gamma \epsilon_{x}=\frac{C_{q} \gamma^{3}}{12\left(J_{x 0}+F_{w}\right.}\left[\frac{\epsilon_{r} \theta^{3}}{\sqrt{15}}+\frac{F_{w}\left|B_{w}^{3}\right| \lambda_{w}^{2}<\beta_{x}>_{w}}{16(B \rho)^{3}}\right],
$$

where

$$
F_{w} \approx \frac{L_{w} B_{w}^{2}}{4 \pi(B \rho) B_{a}}
$$

and $\theta=B_{a} L_{a} /(B \rho)$.

First, the wiggler period $\lambda_{w}$ was reduced from $40 \mathrm{~cm}$ to $27 \mathrm{~mm}$, which is the period of the NLC wiggler design [5]. As a next step, using Eq. (1), $\gamma \epsilon_{x}$ was computed as a function of the arc and wiggler fields $B_{a}$ and $B_{w}$. The arc bending field was next decreased from $1.94 \mathrm{kG}$ to 1.535 $\mathrm{kG}$, and at the same time the total number of arc cells increased from 69 to 76, thereby reducing $\theta$ in (1). Equation (1) suggests that decreasing $B_{w}$ would further reduce the emittance. However, this is not the case at the design bunch population of $2 \times 10^{10}$, if intrabeam scattering is taken into account (see Fig. 4 below). Therefore, the wiggler field was left at $21.5 \mathrm{kG}$. The length of a wiggler consisting of 8 periods is about $2 \mathrm{~m}$, matching the FODO cell length in the straight sections. The optics between wiggler sections and arcs was rematched.

The rf frequency was left at the original value of 714 $\mathrm{MHz}$ (the same frequency as in the SLC damping rings or the ATF), as increasing the frequency by a factor of 2 aggravates the effect of intrabeam scattering. Different beam energies were not studied.

In Table 1 the original parameters for the "TME5.1" design are compared with those obtained after modifying the wigglers or both the wigglers and the arcs, respectively. Figure 1 shows the horizontal beta function around the ring, which gives an impression of the overall layout.

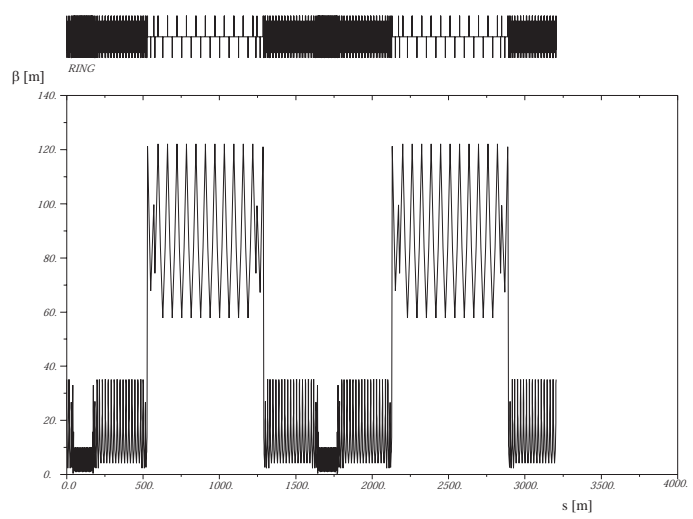

Figure 1: Horizontal beta function around the 3-km damping ring. 
Table 1: Parameters of three ILC damping-ring designs with 3-km circumference

\begin{tabular}{|l|ccc|}
\hline Parameter & TME5.1 & with NLC wiggler & $\begin{array}{c}\text { with NLC wiggler } \\
\text { and modified arcs }\end{array}$ \\
\hline Ring energy [GeV] & 5.0 & 5.0 & 5.0 \\
\hline Ring circumference [m] & 3223.8 & 3223.8 & 3201.0 \\
\hline No. of bunch trains stored & 60 & 60 & 31 \\
\hline No. of bunches/trains stored & 43 & 43 & 100 \\
\hline Train spacing [ns] & 61 & 61 & 67 \\
\hline Bunch spacing [ns] & 2.8 & 2.8 & 2.8 \\
\hline Bunch population & $1.4 \times 10^{10}$ & $1.4 \times 10^{10}$ & $2.0 \times 10^{10}$ \\
\hline Horizontal emittance (norm) [nm] & 3892 & 2841 & $2030(2200)$ \\
\hline rms energy spread [\%] & 0.136 & 0.15 & 0.151 \\
\hline rms bunch length [mm] & 7.37 & 9.94 & 9.6 \\
\hline Damping time x/y/z [msec] & $12.1 / 12.1 / 6.08$ & $8.1 / 8.1 / 4.05$ & $8.12 / 8.12 / 4.6$ \\
\hline Betatron tune x/y & $45.36 / 24.55$ & $45.36 / 24.78$ & $48.85 / 27.19$ \\
\hline Number of cells & 60 & 60 & 76 \\
\hline Field of bending magnet [T] & 0.194 & 0.194 & 0.153 \\
\hline Length of bending magnet [m] & 9 & 9 & 9 \\
\hline Number of wigglers & 80 & 80 & 80 \\
\hline Wiggler period [cm] & 40 & 27 & 27 \\
\hline Field of wiggler [T] & 1.8 & 2.15 & 2.15 \\
\hline Energy loss per turn [MeV] & 8.85 & 13.28 & 13.15 \\
\hline RF frequency [MHz] & 714 & 714 & 714 \\
\hline Effective RF voltage [MV] & 16 & 16 & 16 \\
\hline Momentum compaction & $3.6 \times 10^{-4}$ & $3.6 \times 10^{-4}$ & $3.5 \times 10^{-4}$ \\
\hline
\end{tabular}

\section{PERFORMANCE}

The performance evaluation takes into account the effect of radiation damping, quantum excitation, and intrabeam scattering, as described for the CLIC damping ring design in $[7,8]$. The intrabeam scattering contribution is computed using the "modified Piwinski formalism" $[9,10]$. Figures 2 and 3 present the emittance evolution after injection, assuming injected beam emittances of $\gamma \epsilon_{x} \approx 120 \mu \mathrm{m}$ and $\gamma \epsilon_{y} \approx 60 \mu \mathrm{m}$. The contribution from intrabeam scattering is not neglibible. Nevertheless, this ring achieves a final horizontal emittance of $2 \mu \mathrm{m}$, which is a factor four below the ILC target value and factor two improvement compared with "TME5.1".

Figure 4 compares the horizontal equilibrium emittances for wiggler fields of $21.5 \mathrm{kG}$ and $15.6 \mathrm{kG}$. At bunch populations above $N_{b} \approx 1.7 \times 10^{10}$, the higher wiggler field yields a smaller emittance. For the stronger field, the equilibrium bunch length is about $50 \%$ higher, independent of the bunch population, as is illustrated in Fig. 5.

Figure 6 displays the on-momentum dynamic aperture obtained by tracking. The horizontal and vertical amplitudes on the two axes are expressed in terms of the final rms beam sizes. The maximum beam emittances which could be injected into the dynamic aperture of this ring are about $\gamma \epsilon_{x} \approx 1000 \mu \mathrm{m}$ and $\gamma \epsilon_{y} \approx 60 \mu \mathrm{m}$. Figure 7 illustrates the nonlinear chromaticity.

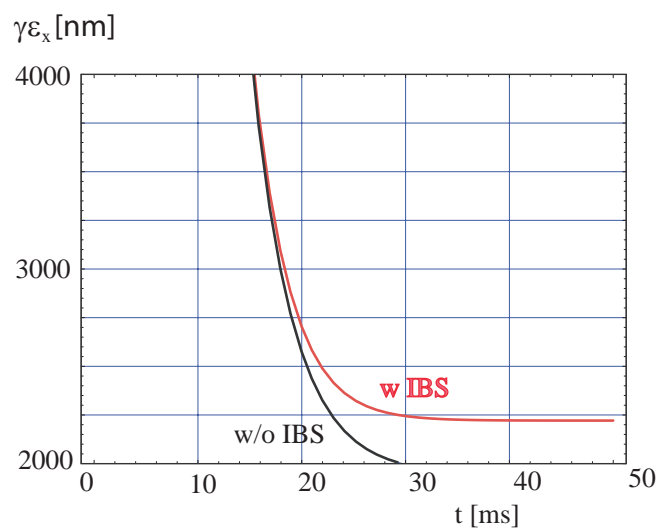

Figure 2: Normalized horizontal emittance as a function of time computed with and without intrabeam scattering.

Intrabeam scattering was included in all emittance calculations presented here. The importance of other collective effects can be estimated as for CLIC in Ref. [11].

\section{CONCLUSION}

The 3-km damping ring presented here provides an emittance of $\gamma \epsilon_{x} \approx 2 \mu \mathrm{m}$, i.e., 4 times smaller than the ILC target, and it has a circumference of about $3 \mathrm{~km}$. The horizontal dynamic aperture is sufficiently large to accept a beam 
$\gamma \varepsilon_{\mathrm{y}}[\mathrm{nm}]$

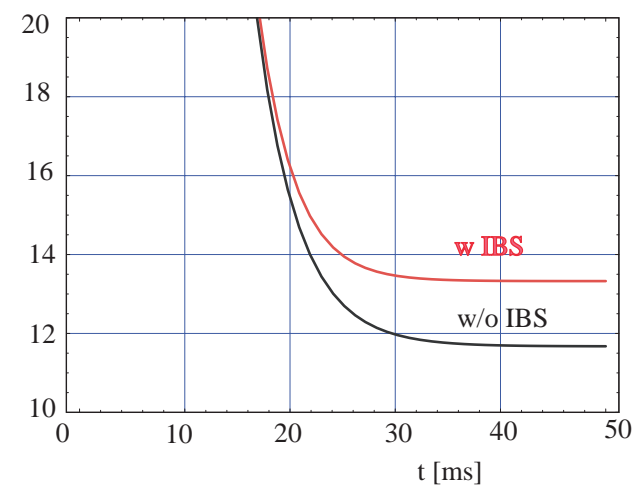

Figure 3: Normalized vertical emittance as a function of time computed with and without intrabeam scattering; and assuming $0.6 \%$ betatron coupling.

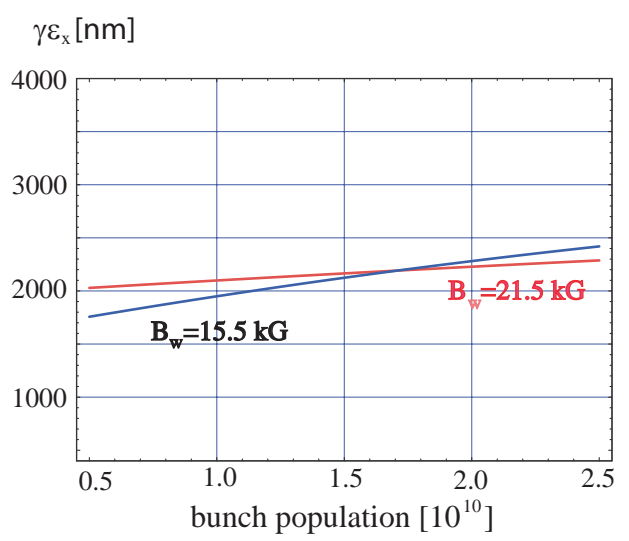

Figure 4: Horizontal equilibrium emittance as a function of bunch population with intrabeam scattering for two different values of the wiggler field, assuming $0.6 \%$ betatron coupling.

of $\gamma \epsilon_{x 0} \approx 0.01 \mathrm{~m}$. The vertical acceptance corresponds to about $60 \mu \mathrm{m}$. Further optimization is possible.

\section{REFERENCES}

[1] W. Decking, "Optical Layout for the TESLA 5-GeV Damping Ring,” DESY-TESLA-2001-11 (2001).

[2] L. Emery et al., "Studies Pertaining to a Small Damping Ring for the Inernational Linear Collider," FERMILAB-TM-2272AD-TD (2004).

[3] J. Urakawa, "Alternative DR Designs - Overview," First ILC KEK Meeting, November 14, 2004.

[4] P. Emma, T. Raubenheimer, "Systematic Approach to Damping Ring Design,” PRST-AB 4, 021001 (2001).

[5] J. Corlett et al., "NLC Damping Ring Wiggler Studies 1999," LCC-0031 (1999); J. Corlett, S. Marks, M.C. Ross, "Transverse Field Profile of the NLC Damping Rings Electronmagnet Wiggler," LCC-0038, CBP Tech-Note-234 (2000).

[6] W. Decking, R. Brinkmann, EPAC 2000 Vienna (2000).

[7] M. Korostelev, F. Zimmermann, "Optimization of CLIC Damping Ring Design Parameters," EPAC'02 Paris (2002).

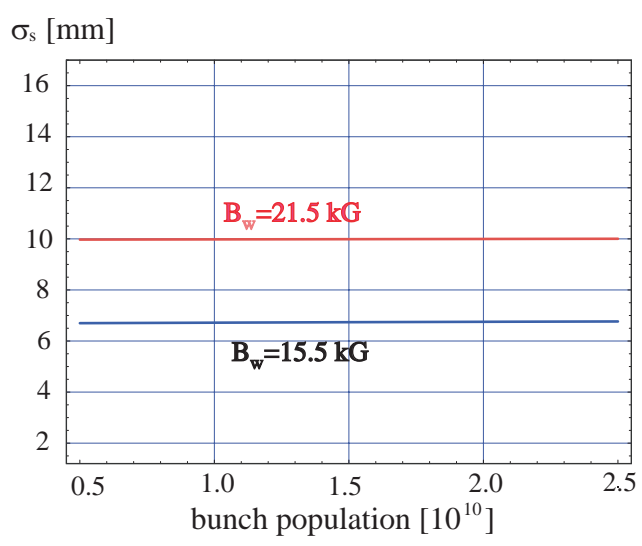

Figure 5: Equilibrium rms bunch length as a function of bunch population with intrabeam scattering for two different values of the wiggler field, assuming $0.6 \%$ betatron coupling.

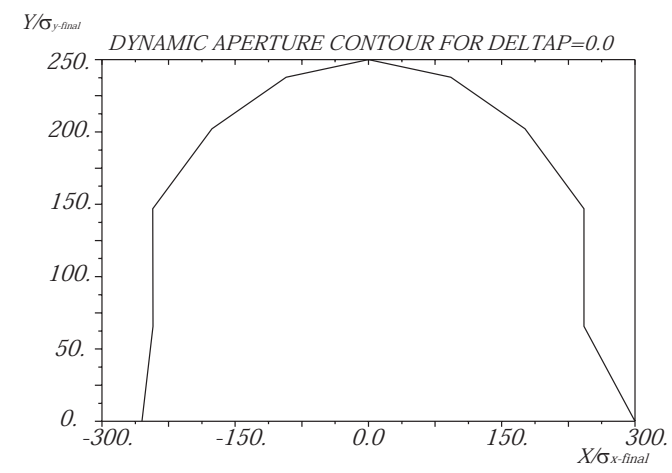

Figure 6: Dynamic aperture in units of the final beam sizes.

[8] M. Korostelev, F. Zimmermann, Proc. Nanobeam'02 Lausanne, CERN-Proceedings-2003-001 (2001).

[9] A. Piwinski, 9th HEACC'74 Stanford (1974).

[10] K.L.F. Bane, EPAC'02 Paris (2002).

[11] F. Zimmermann et al., "Collective Effects in the CLIC Damping Rings," these proceedings.

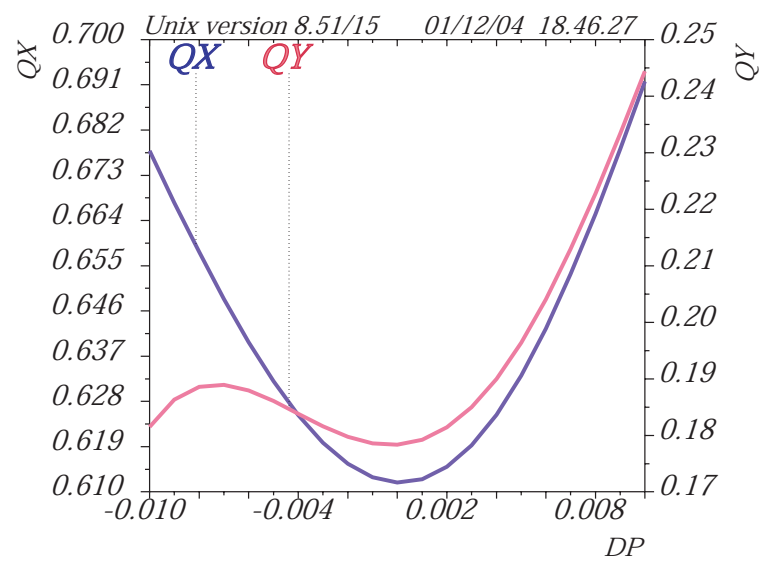

Figure 7: Nonlinear chromaticity. 